Article

\title{
Prediction of Partition Coefficients of Organic Compounds between SPME/PDMS and Aqueous Solution
}

\author{
Keh-Ping Chao *, Yu-Ting Lu and Hsiu-Wen Yang \\ Department of Occupational Safety and Health, China Medical University, 91 Hsueh-Shih Rd., \\ Taichung 40402, Taiwan; E-Mails: disney-14014@hotmail.com (Y.-T.L.); \\ hwyang1224@gmail.com (H.-W.Y.) \\ * Author to whom correspondence should be addressed; E-Mail: kpchao@mail.cmu.edu.tw; \\ Tel.: +886-4-2205-3366 (ext. 6205); Fax: +886-4-2207-0500.
}

Received: 3 January 2014; in revised form: 6 February 2014 / Accepted: 11 February 2014 / Published: 14 February 2014

\begin{abstract}
Polydimethylsiloxane (PDMS) is commonly used as the coated polymer in the solid phase microextraction (SPME) technique. In this study, the partition coefficients of organic compounds between SPME/PDMS and the aqueous solution were compiled from the literature sources. The correlation analysis for partition coefficients was conducted to interpret the effect of their physicochemical properties and descriptors on the partitioning process. The PDMS-water partition coefficients were significantly correlated to the polarizability of organic compounds $(r=0.977, p<0.05)$. An empirical model, consisting of the polarizability, the molecular connectivity index, and an indicator variable, was developed to appropriately predict the partition coefficients of 61 organic compounds for the training set. The predictive ability of the empirical model was demonstrated by using it on a test set of 26 chemicals not included in the training set. The empirical model, applying the straightforward calculated molecular descriptors, for estimating the PDMS-water partition coefficient will contribute to the practical applications of the SPME technique.
\end{abstract}

Keywords: polydimethylsiloxane (PDMS); solid phase microextraction (SPME); partition coefficient; polarizability; molecular connectivity index 


\section{Introduction}

Solid phase microextraction (SPME) is a solvent-free sample preparation technique. The outer layer of the fused silica rod of the SPME device is coated with polymeric materials, such as polydimethylsiloxane. When the SPME device is placed in a sample matrix, the analyte is extracted and sorbed from the sample matrix onto the polymeric coating stationary phase. The analyte is then desorbed from the SPME device and the concentrated extract is analyzed using an instrument such as a gas chromatograph. The SPME technique simplifies the four steps of sampling, extraction, condensation and introduction of the sample into the analytical instrument [1]. In comparison to the traditional solid phase extraction methods, the advantages of SPME are lower cost, easy handling and shorter time procedures. Therefore, SPME has been widely used in the analysis of many organic compounds in water [2,3].

Sorption of the analyte between the sample matrix and the coating phase may be assumed to be an equilibrium partitioning process and may be modeled by a linear isotherm as follows:

$$
K_{f s}=\frac{C_{f}}{C_{s}}
$$

where $C_{f}$ and $C_{s}$ are the equilibrium concentrations of the analyte in the coating and the sample matrix, respectively $\left(\mathrm{M} / \mathrm{L}^{3}\right)$; and $K_{f s}$ is the coating-matrix partition coefficient.

For an aqueous matrix, the total mass of analyte in the coating and the sample matrix can be represented as:

$$
C_{o} V_{s}=C_{f} V_{f}+C_{s} V_{s}=\left(K_{f s} V_{f}+V_{s}\right) C_{s}
$$

where $C_{\mathrm{o}}$ is the initial concentrations of the analyte in the sample matrix $\left(\mathrm{M} / \mathrm{L}^{3}\right)$; and $V_{f}$ and $V_{s}$ are the volumes of the coating and the sample matrix, respectively $\left(\mathrm{L}^{3}\right)$.

The mass of analyte sorbed by the coating of SPME, $M_{f}(\mathrm{M})$, can be determined as:

$$
M_{f}=C_{f} V_{f}=K_{f s} C_{s} V_{f}=\frac{K_{f s} C_{o} V_{s} V_{f}}{K_{f s} V_{f}+V_{s}}
$$

Prior to using the SPME technique for sampling and analysis, the first step is to select the appropriate polymer as the SPME coated material to sorb the analyte. As shown in Equation 3, the mass of analyte sorbed by SPME is determined by the coating-matrix partition coefficient $K_{f s}$. The proper application of SPME is primarily dependent on the partition coefficient of analyte between the polymeric coating and sample matrix. Therefore, there is a need to estimate the partition coefficient $K_{f s}$ for determining the sensitivity of SPME extraction [4-6].

Factors affecting the sorption of organic compounds onto the solid phase include electrical attraction, chemical affinity of the organic molecule, van der Waal's force and the hydrophobic nature of the organic compound [7]. The physicochemical descriptors, such as polarizability, molecular connectivity index, and indicator variable, have been found to be successful in modeling the parameters for partition processes like octanol-water, octanol-air and air-water partition coefficients as well as adsorption coefficients and aqueous solubility of organic compounds [8-12]. 
This study is an attempt to develop predictive equations for the partition coefficients of a variety of chemicals in aqueous solutions onto SPME/PDMS based on their physicochemical properties and molecular structures. This was achieved by using the PDMS-water partition coefficients, $K_{f w}$, retrieved from the literature $[13,14]$ and relating them to their physicochemical descriptors, such as polarizability $(\Phi)$, molecular connectivity index $\left({ }^{1} \chi\right)$, indicator variable $(I)$, water solubility $(W S)$, molecular weight $\left(M_{\mathrm{W}}\right)$, and octanol-water partition coefficient $\left(\log K_{\mathrm{ow}}\right)$. The correlation analysis of $K_{f w}$ can provide an understanding in the partitioning process between SPME/PDMS and aqueous samples. Further, the predictive equations will provide a means of determination $K_{f s}$ for other chemicals, and facilitate the application of the SPME technique.

\section{Results and Discussion}

\subsection{Correlation Analysis}

PDMS is a hydrophobic and nonpolar polymer. Therefore, the nonpolar attractive force causing physical adsorption of organic compounds onto PDMS surfaces is the van der Waals force. One of the attractive potential energies for the van der Waals forces is the dipole-induced dipole interaction, also called London dispersion force, between PDMS and organic molecules. When SPME/PDMS is placed in the aqueous solution, the dipole moment of PDMS creates an electric field which polarizes the charges on organic molecules. The magnitude of induced change in dipole moment is determined by the polarizability of organic molecules [15]. As presented in Table $1, K_{f w}$ was found to correlate best with the polarizability of organic compounds $(r=0.977, p<0.05)$. Therefore, the polarizability can be a good basis to understand the partition between SPME/PDMS and organic compounds in the aqueous solution.

Table 1. Correlation analysis of $K_{f w}$ for the training set.

\begin{tabular}{cc}
\hline Descriptor & Correlation \\
\hline$\Phi$ & $\log K_{f w}=1.933+1.026 \Phi(r=0.977, p<0.05)$ \\
$\log K_{\text {ow }}$ & $\log K_{f w}=0.319+0.881 \log K_{\text {ow }}(r=0.774, p<0.05)$ \\
$1 \chi$ & $\log K_{f w}=0.750+0.862^{1} \chi(r=0.743, p<0.05)$ \\
$I$ & $\log K_{f w}=4.144-0.846 I(r=0.583, p<0.05)$ \\
$W S$ & $\log K_{f w}=3.936-0.002 W S(r=0.559, p<0.05)$ \\
$M_{\mathrm{W}}$ & $\log K_{f w}=1.859+0.017 M_{\mathrm{W}}(r=0.474, p<0.05)$ \\
\hline
\end{tabular}

In general, the van der Waals forces of molecules increase with an increase in their molecular weights [7]. Table 1 shows that $K_{f w}$ was proportional to the molecular weights of organic compounds but only with a correlation coefficient $r=0.474(p<0.05)$. If the organic compounds were grouped in different categories, i.e., alkanes and aromatic hydrocarbons, for correlation analysis, Figure 1 indicates that $K_{f w}$ was significantly correlated to their molecular weights $(r \geq 0.916)$. This result may imply that diffusion of organic molecules in the PDMS fiber was not a governing mechanism for the mass transfer between PDMS and organic compounds. 
Figure 1. Correlations of $K_{f w}$ for the training set with their molecular weights.

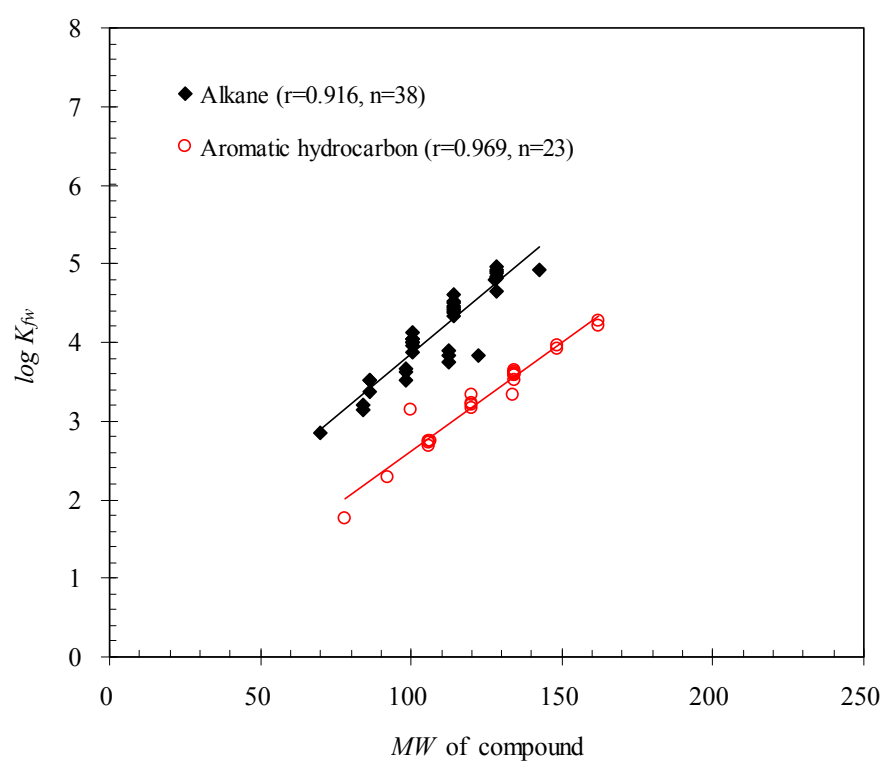

According to the principle of "like dissolves like", it is plausible that a non-polar compound is more easily sorbed by PDMS. In general, the larger the octanol-water partition coefficient of a chemical, the lower the polarity. Several researchers indicated that $\log K_{f w}$ of SPME/PDMS linearly increased with $\log K_{\text {ow }}$ for organic compounds [16,17]. As seen in Table $1, \log K_{f w}$ for the training set showed a positive correlation with their $\log K_{\text {ow }}(r=0.774, p<0.05)$. On the other hand, an inversely proportional trend was observed between $\log K_{f w}$ and water solubility $(r=0.559, p<0.05)$, indicating that the less soluble compound is more likely partitioned to SPME/PDMS than more soluble compounds.

The molecular connectivity index conveys the degree of branching for the molecular structure [18]. The greater the degree of branching in a molecule, the lower will be the value of the molecular connectivity index. Table 1 shows that $K_{f w}$ was well proportional to their molecular connectivity indexes with a correlation coefficient $r=0.743(p<0.05)$. Several researchers have indicated that the molecular connectivity indexes were significantly correlated to polarizability $[12,18]$. In this study, the molecular connectivity indexes of the training set were proportional to their polarizability $(r=0.676$, $p<0.05$ ). However, it is speculated that the branch molecule is less easily partitioned to SPME/PDMS.

\subsection{Empirical Models}

The best predictive model for $K_{f w}$ was obtained through the multiple regression analysis using a statistical software package SPSS 20.0 version (IBM, New York, NY, USA, 2011). Based on the stepwise regression with $95 \%$ confidence limits, the multiple regression analyses of $K_{f w}$ was as follows $(r=0.99, p<0.05)$ :

$$
\log K_{f w}=1.545+0.724 \Phi+0.292{ }^{1} \chi-0.227 I+0.001 W S
$$

The above model explained $98 \%$ of the variance in the $K_{f w}$ data of the training set. Nirmalakhandan and Speece [12] developed a quantitative structure-activity relationship (QSAR) model for estimating Henry's constant. In their study, the best predictive equation consisted of the molecular connectivity 
index, polarizability, and indicator variable of organic compounds. For the physicochemical descriptors of Equation (4), Table 1 indicates that the water solubility $(r=0.559)$ and the indicator variable $(r=0.583)$ were correlated to $K_{f w}$ with the lower correlation coefficients. If the water solubility and the indicator variable were excluded for Equation (4), the multiple regressions of $K_{f w}$ were as follows:

$$
\begin{gathered}
\log K_{f w}=1.447+0.728 \Phi+0.316^{1} \chi-0.224 I \\
\log K_{f w}=1.502+0.918 \Phi+0.177^{1} \chi
\end{gathered}
$$

For Equations (5) and (6), the correlation coefficients $(r)$ were 0.989 and 0.984 , respectively, which were similar to that of Equation (4). Figure 2 presents a comparison of $K_{f w}$ for the training set with $K_{f w}$ predicted from Equations (4)-(6). Figure 2 shows that the agreement between the training set and the models predicted $K_{f w}$ is very satisfactory.

Figure 2. Comparison of $K_{f w}$ between the training set and predictive models.

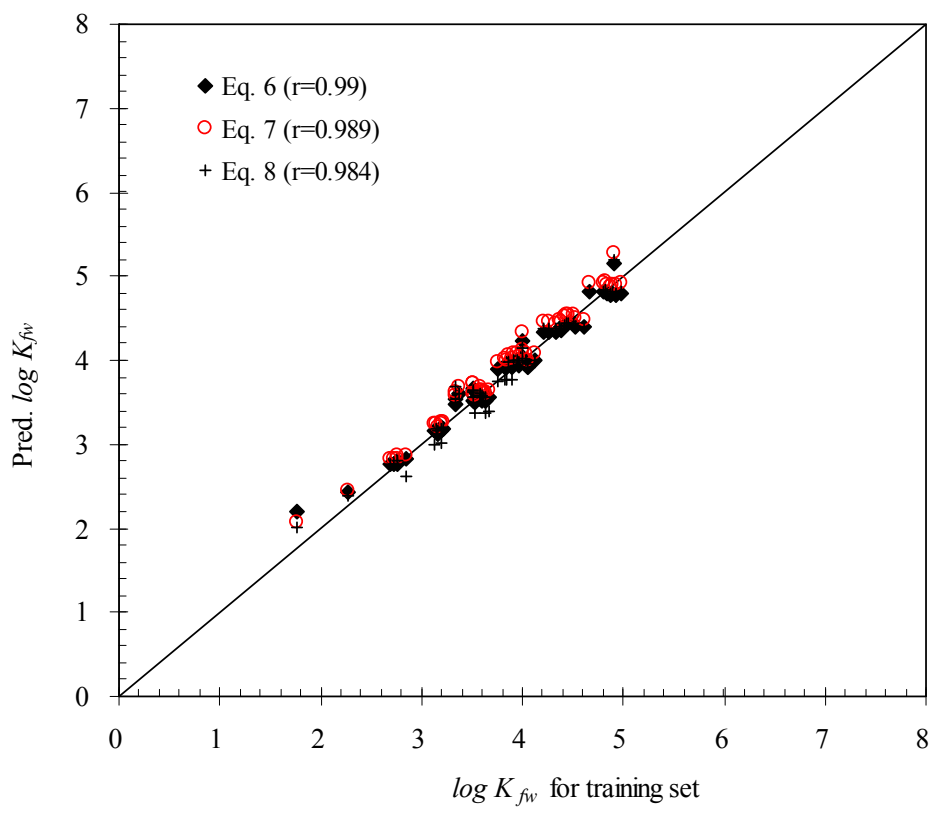

Several researchers have used the linear solvation energy relationship (LSER) method to estimate $K_{f w}$ for SPME [19,20]. The LSER model consists of five descriptors of chemicals, i.e., molar refraction, polarizability, hydrogen bond acidity, hydrogen bond basicity, and McGowan characteristic volume (mainly representing London dispersion). As compared with the models developed herein, the physicochemical descriptors for the LSER method require a large data set. In particular, the physicochemical property data for the LSER method may be unavailable for the compound of interest $[16,21]$.

To assess the validity of the above empirical models, $K_{f w}$ for the organic compounds of the test set was predicted using Equations (4)-(6). Figure 3 indicates a comparison of $K_{f w}$ for the test set with $K_{f w}$ predicted using the empirical models. As shown in Figure 3, most of $K_{f w}$ for the test set were greater than those predicted using the empirical models. The slopes of regression analysis shown in Figure 3 implied that there was a systematic difference in the experimental measurement of $K_{f w}$ between the training and test sets. For example, Table 3 indicates that the values of $K_{f w}$ were 566 and 564 for 
ethylbenzene and $p$-xylene, respectively. In the study of Xia et al. [14], $K_{f w}$ was 512 and 575 for ethylbenzene and $p$-xylene which were not included in the test set. However, the correlation coefficients of $K_{f w}$ were $0.813,0.819$ and 0.771 for Equations (4)-(6), respectively. For a practical purpose, the regression equation, consisting of the molecular connectivity index, polarizability, and indicator variable, can be an adequate model to predict $K_{f w}$ for SPME/PDMS.

Figure 3. Comparison of $K_{f w}$ between the test set and predictive models.

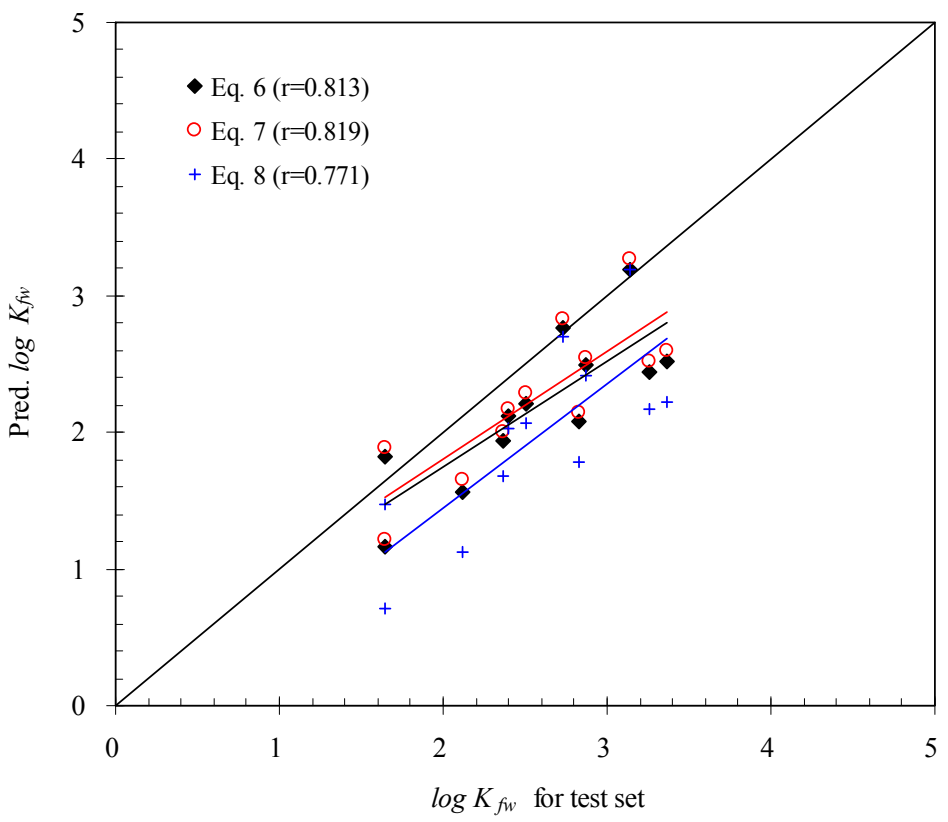

Table 2. The training set of PDMS-water partition coefficients and physico-chemical descriptors.

\begin{tabular}{cccccccc}
\hline Compound & $\boldsymbol{K}_{f w}$ & ${ }^{1} \boldsymbol{\chi}$ & $\boldsymbol{\Phi}$ & $\boldsymbol{I}$ & $\boldsymbol{M}_{\mathbf{W}}^{\dagger}$ & $\boldsymbol{W S}^{*}$ & $\boldsymbol{l o g}_{\boldsymbol{~}} \boldsymbol{K}_{\mathbf{w}}$ \\
\hline Benzene & 58 & 2 & 0.165 & 1 & 78 & 1790 & 2.13 \\
Toluene & 189 & 2.411 & 0.502 & 1 & 92 & 526 & 2.73 \\
$o$-Xylene & 485 & 2.827 & 0.839 & 1 & 106 & 178 & 3.12 \\
$m$-Xylene & 533 & 2.821 & 0.839 & 1 & 106 & 161 & 3.2 \\
$p$-Xylene & 564 & 2.821 & 0.839 & 1 & 106 & 162 & 3.15 \\
Ethylbenzene & 566 & 2.971 & 0.839 & 1 & 106 & 169 & 3.15 \\
Cyclopentane & 712 & 2.5 & 0.733 & 0 & 70 & 156 & 3.01 \\
Methylcyclopentane & 1356 & 2.894 & 1.07 & 0 & 84 & 42 & 3.37 \\
Isopropylbenzene & 1412 & 3.354 & 1.176 & 1 & 120 & 61.3 & 3.66 \\
1,3,5-Trimethylbenzene & 1451 & 3.232 & 1.176 & 1 & 120 & 48.2 & 3.42 \\
1-Methyl-4-Ethylbenzene & 1581 & 3.382 & 1.176 & 1 & 120 & 94.9 & 3.63 \\
Cyclohexane & 1592 & 3 & 1.07 & 0 & 84 & 55 & 3.44 \\
n-Propylbenzene & 1664 & 3.471 & 1.176 & 1 & 120 & 52.2 & 3.69 \\
1,2,4-Trimethylbenzene & 2183 & 3.238 & 1.76 & 1 & 120 & 57 & 3.63 \\
tert -Butylbenzene & 2185 & 3.661 & 1.513 & 1 & 134 & 29.5 & 4.11 \\
2,3-Dimethylbutane & 2359 & 2.643 & 1.782 & 0 & 86 & 22.5 & 3.42 \\
2-Methylpentane & 3224 & 2.77 & 1.782 & 0 & 86 & 14 & 3.21 \\
3-Methylpentane & 3270 & 2.808 & 1.782 & 0 & 86 & 17.9 & 3.6 \\
\hline
\end{tabular}


Table 2. Cont.

\begin{tabular}{|c|c|c|c|c|c|c|c|}
\hline Compound & $K_{f w}$ & ${ }^{1} \chi$ & $\Phi$ & $I$ & $\boldsymbol{M}_{\mathbf{W}}^{\dagger}$ & $W S^{*}$ & $\log K_{\mathrm{ow}}$ \\
\hline 1-Methyl-3-Isopropylbenzene & 3284 & 3.765 & 1.513 & 1 & 134 & 42.5 & 4.5 \\
\hline trans-1,2-Dimethylcyclopentane & 3372 & 3.207 & 1.407 & 0 & 98 & 33.9 & 3.52 \\
\hline 1-Methyl-3-n-Propylbenzene & 3772 & 3.882 & 1.513 & 1 & 134 & 9.09 & 4.67 \\
\hline$n$-Butylbenzene & 3872 & 3.971 & 1.513 & 1 & 134 & 11.8 & 4.38 \\
\hline 1,2-Dimethyl-4-Ethylbenzene & 3984 & 3.8 & 1.513 & 1 & 134 & 12.7 & 4.5 \\
\hline sec-Butylbenzene & 4011 & 3.892 & 1.513 & 1 & 134 & 17.6 & 4.57 \\
\hline Isobutylbenzene & 4197 & 3.827 & 1.513 & 1 & 134 & 10.1 & 4.68 \\
\hline cis-1,3-Dimethylcyclopentane & 4289 & 3.288 & 1.407 & 0 & 98 & 33.9 & 3.52 \\
\hline 1,3-Dimethyl-2-Ethylbenzene & 4345 & 3.805 & 1.513 & 1 & 134 & 19.6 & 4.28 \\
\hline Methylcyclohexane & 4657 & 3.394 & 1.407 & 0 & 98 & 14 & 3.61 \\
\hline $\begin{array}{l}\text { cis-trans-cis-1,2,4- } \\
\text { Trimethylcyclopentane }\end{array}$ & 5621 & 3.698 & 1.744 & 0 & 112 & 14.8 & 3.94 \\
\hline trans-1,2-Dimethylcyclohexane & 6638 & 3.805 & 1.744 & 0 & 112 & 5.2 & 4.01 \\
\hline 1-Ethyl-1-methylcyclopentane & 6831 & 3.768 & 1.744 & 0 & 122 & 11.6 & 4.05 \\
\hline 2,2-Dimethylpentane & 7349 & 3.061 & 2.119 & 0 & 100 & 4.4 & 3.67 \\
\hline cis-1,2-Dimethylcyclohexane & 7826 & 3.805 & 1.744 & 0 & 112 & 6 & 4.01 \\
\hline$n$-Pentylbenzene & 8195 & 4.471 & 1.85 & 1 & 148 & 3.37 & 4.9 \\
\hline 2,4-Dimethylpentane & 8989 & 3.126 & 2.119 & 0 & 100 & 5.5 & 3.63 \\
\hline 2-Methylbutylbenzene & 9099 & 4.365 & 1.85 & 1 & 148 & 12.7 & 4.43 \\
\hline 2,2,3-Trimethylbutane & 9802 & 3.944 & 2.119 & 0 & 100 & 28.9 & 3.59 \\
\hline 2-Methylhexane & 10,202 & 3.27 & 2.119 & 0 & 100 & 2.54 & 3.71 \\
\hline 3,3-Dimethylpentane & 10,963 & 3.121 & 2.119 & 0 & 100 & 5.92 & 3.67 \\
\hline 3-Methylhexane & 11,146 & 2.9 & 2.119 & 0 & 100 & 4.95 & 3.71 \\
\hline 2,3-Dimethylpentane & 13,074 & 3.181 & 2.119 & 0 & 100 & 5.25 & 3.63 \\
\hline 1,2,4-Triethylbenzene & 16,253 & 4.92 & 2.187 & 1 & 162 & 2.9 & 5.11 \\
\hline 1,3,5-Triethylbenzene & 18,517 & 4.914 & 2.187 & 1 & 162 & 0.014 & 5.11 \\
\hline 2,2,3-Trimethylpentane & 21,205 & 3.481 & 2.456 & 0 & 114 & 2.4 & 4.09 \\
\hline 2,5-Dimethylhexane & 23,519 & 3.626 & 2.456 & 0 & 114 & 9.2 & 4.12 \\
\hline 2,2-Dimethylhexane & 24,504 & 3.561 & 2.456 & 0 & 114 & 0.2 & 4.16 \\
\hline 2-Methylheptane & 25,806 & 3.77 & 2.456 & 0 & 114 & 7.97 & 4.2 \\
\hline 4-Methylheptane & 27,274 & 3.808 & 2.456 & 0 & 114 & 7.97 & 4.2 \\
\hline 3-Ethylhexane & 28,370 & 3.846 & 2.456 & 0 & 114 & 7.97 & 4.2 \\
\hline 3-Methylheptane & 31,856 & 3.808 & 2.456 & 0 & 114 & 0.792 & 4.2 \\
\hline 2,3-Dimethylhexane & 33,749 & 3.681 & 2.456 & 0 & 114 & 9.2 & 4.12 \\
\hline 2,4-Dimethylhexane & 41,133 & 3.664 & 2.456 & 0 & 114 & 9.87 & 4.12 \\
\hline 2-Methyloctane & 45,267 & 4.27 & 2.793 & 0 & 128 & 2.87 & 4.69 \\
\hline 3,3-Diethylpentane & 63,718 & 4.243 & 2.793 & 0 & 128 & 2.9 & 4.65 \\
\hline 3-Methyloctane & 66,682 & 4.308 & 2.793 & 0 & 128 & 2.87 & 4.69 \\
\hline 2,3-Dimethylheptane & 68,675 & 4.181 & 2.793 & 0 & 128 & 3.37 & 4.61 \\
\hline 3,3-Dimethylheptane & 76,013 & 4.121 & 2.793 & 0 & 128 & 2.9 & 4.65 \\
\hline 3,5-Dimethylheptane & 78,829 & 4.202 & 2.793 & 0 & 128 & 3.11 & 4.61 \\
\hline 3,3-Dimethyloctane & 82,430 & 4.621 & 3.13 & 0 & 142 & 2.9 & 5.14 \\
\hline 2,5-Dimethylheptane & 84,142 & 4.164 & 2.793 & 0 & 128 & 3.11 & 4.61 \\
\hline 3,4-Dimethylheptane & 93,292 & 4.219 & 2.793 & 0 & 128 & 3.11 & 4.61 \\
\hline
\end{tabular}


Table 3. The test set of PDMS-water partition coefficients.

\begin{tabular}{cccccc}
\hline Compound & $\boldsymbol{K}_{f w}$ & ${ }^{1} \boldsymbol{\chi}$ & $\boldsymbol{\Phi}$ & $\boldsymbol{I}$ & $\boldsymbol{W \boldsymbol { S } ^ { \ddagger }}$ \\
\hline Benzyl alcohol & 0.447 & 2.580 & -0.323 & 1 & $4.29 \times 10^{4}$ \\
4-Fluorophenol & 0.525 & 2.234 & -1.110 & 1 & $1.25 \times 10^{4}$ \\
Phenol & 0.661 & 2.134 & -0.540 & 1 & $8.28 \times 10^{4}$ \\
m-Cresol & 0.933 & 2.545 & -0.323 & 1 & $2.27 \times 10^{4}$ \\
Phenethyl alcohol & 1.318 & 3.081 & 0.014 & 1 & $2 \times 10^{4}$ \\
3-Methylbenzyl alcohol & 1.479 & 2.991 & 0.014 & 1 & $5 \times 10^{4}$ \\
3-Chlorophenol & 2.042 & 2.647 & -0.727 & 1 & $2.6 \times 10^{4}$ \\
3,5-Dimethylphenol & 2.630 & 2.956 & 0.014 & 1 & $4.88 \times 10^{3}$ \\
3-Bromophenol & 2.884 & 3.026 & -0.762 & 1 & $2.4 \times 10^{4}$ \\
4-Ethylphenol & 3.981 & 3.106 & 0.014 & 1 & $4.9 \times 10^{3}$ \\
4-Chloroaniline & 6.918 & 2.676 & -0.022 & 1 & $3.9 \times 10^{3}$ \\
Phenyl acetate & 7.244 & 3.023 & -1.430 & 1 & $4.64 \times 10^{3}$ \\
Benzonitrile & 10.96 & 2.384 & 0.753 & 1 & $2 \times 10^{3}$ \\
Acetophenone & 10.96 & 2.865 & -0.605 & 1 & $6.13 \times 10^{3}$ \\
4-Chloroacetophenone & 43.7 & 3.342 & -0.672 & 1 & 111 \\
Methyl benzoate & 44.7 & 2.977 & -1.430 & 1 & $2.1 \times 10^{3}$ \\
Ethylbenzoate & 131.8 & 3.565 & -1.093 & 1 & 720 \\
4-Chloroanisole & 234.4 & 3.036 & -0.390 & 1 & 237 \\
Chlorobenzene & 251.2 & 2.477 & 0.098 & 1 & 498 \\
Bromobenzene & 323.6 & 2.891 & 0.063 & 1 & 410 \\
Iodobenzene & 537.1 & 3.161 & 0.692 & 1 & 340 \\
Naphthalene & 676.1 & 3.405 & -0.344 & 1 & 31 \\
4-Chlorotoluene & 741.3 & 3.095 & 0.435 & 1 & 106 \\
Propylbenzene & 1380 & 3.471 & 1.176 & 1 & 52.2 \\
1-Methylnaphthalene & 1819 & 3.821 & -0.007 & 1 & 25.8 \\
Biphenyl & 2344 & 4.071 & -0.007 & 1 & 6.9 \\
\hline
\end{tabular}

${ }^{\ddagger}$ WS: $\mathrm{mg} / \mathrm{L}$.

\section{Method}

\subsection{PDMS-Water Partition Coefficients}

The training set of PDMS-water partition coefficients for alkanes and aromatic hydrocarbons was compiled from the literature [13] published by Dr. Pawliszyn who invented the SPME technique. As shown in Table 2, the values of $K_{f w}(n=61)$ exhibit a wide range from 58 (for benzene) to 82,430 (for 3,3-dimethyloctane). In addition, the organic compounds of the training set have a wide range of water solubility, e.g., $1.4 \times 10^{-2} \mathrm{mg} / \mathrm{L}$ for $1,3,5$-triethylbenzene, and $1.79 \times 10^{3} \mathrm{mg} / \mathrm{L}$ for benzene. The test set of PDMS-water partition coefficients $(n=26)$, not included in the training set, was retrieved from the study of Xia et al. [14]. Table 3 indicates that the magnitude of $K_{f w}$ values for the test set, consisting of alcohols, benzenes and phenols, were between $10^{-1}$ and $10^{3} . K_{f w}$ for the training and test sets were experimentally obtained using the SPME/PDMS devices at $25^{\circ} \mathrm{C}$. 


\subsection{Physicochemical Descriptors}

In this study, the polarizability and molecular connectivity index were used to represent the interactions between the analyte and PDMS coating. As shown in Equation (7), the polarizability $\Phi$ was determined by the addition of atom/bond contribution factors [12,22].

$$
\Phi=\sum F_{i} \times(\text { number of atom } / \text { bond })
$$

where $F_{i}$ is the contribution factors indicated in Table 4 .

Table 4. Contribution factors for the calculation of polarizability.

\begin{tabular}{cccc}
\hline Atom/bond & Contribution factor & Atom/bond & Contribution factor \\
\hline Carbon & 0.577 & Iodine & 0.407 \\
Hydrogen $*$ & -0.120 & Fluorine & -0.570 \\
Oxygen & -0.825 & Cycle & -0.952 \\
Hydroxyl & -3.701 & Double bond & -0.859 \\
Chlorine & -0.187 & Triple bond & -0.109 \\
Bromine & -0.222 & - & - \\
\hline
\end{tabular}

Data was obtained from Nirmalakhandan and Speece [12]; * Attached to carbon atoms only.

The molecular connectivity index is a topological descriptor of molecular structure based on a count of skeletal atom groupings of a chemical compound [18,23]. For each atom of a molecule, a $\delta$ value is assigned as the difference between the number of valence electrons and the number of hydrogen atoms attached to that atom. In this study, the first-order connectivity index, ${ }^{1} \chi$, was calculated as follows $[9,11,12]$ :

$$
{ }^{1} \chi=\sum_{q=1}^{n}\left(\delta_{i} \delta_{j}\right)_{q}^{-0.5}
$$

where $\delta_{i}$ and $\delta_{j}$ are the $\delta$ values of two adjacent atoms $i$ and $j$, respectively; and $n$ is the number of bonds in the molecule.

An indicator variable, $I$, was used to differentiate between compounds on the basis of their ability to take place in hydrogen bonding [24]. I was assigned a value of 1 for all compounds containing an electronegative element, such as oxygen, nitrogen, and halogen etc., attached directly to a carbon atom holding a hydrogen atom. In addition, the value of $I$ was assigned 1 for acetylinic compounds and aromatic compounds with partially substituted hydrogen atoms. For the other compounds, $I$ was set equal to zero [12].

\section{Conclusions}

From the single-parameter model analyses of the training set, the PDMS-water partition coefficients were proportional to the molecular connectivity index, polarizability, molecular weight, and octanol-water partition coefficient of organic compounds, while an inversely proportional trend was observed between $K_{f w}$ and the water solubility as well as the indicator variable. $K_{f w}$ was significantly dependent on the polarizability of the organic compounds. Based on the results of multiple regression analyses, several correlations for $K_{f w}$ were developed using the polarizability, molecular connectivity 
index, indicator variable and water solubility, indicating a good agreement $(r \geq 0.771)$ between the training set and the predicted $K_{f w}$. In addition, the correlations were able to adequately predict $K_{f w}$ for a test set which were not included in the correlation development. The empirical model developed in this work is more versatile than current available correlations. In order to broaden the predictive abilities, however, future work should be conducted to calculate $K_{f w}$ using the empirical model for a variety of organic compounds.

\section{Conflicts of Interest}

The authors declare no conflict of interest.

\section{References}

1. Pawliszyn, J. Applications of Solid Phase Microextraction; Royal Society of Chemistry: Cornwall, UK, 1999.

2. Chao, K.P.; Wang, V.S.; Yang, H.W.; Wang, C.I. Estimation of effective diffusion coefficients for benzene and toluene in PDMS for direct solid phase microextraction. Polym. Test. 2011, 30, 501-508.

3. Mahugo Santana, C.; Sosa Ferrera, Z.; Esther Torres Padrón, M.; Juan Santana Rodríguez, J. Methodologies for the extraction of phenolic compounds from environmental samples: New approaches. Molecules 2009, 14, 298-320.

4. Benhabib, K.; Mimanne, G. Optimized parameters of SMPE analysis for atrazine and its application to measure speciation. Appl. Clay Sci. 2014, 87, 260-264.

5. Benhabib, K.; ter Laak, T.L.; van Leeuwen, H.P. Steady-state diffusion regime in solid-phase microextraction kinetics. Anal. Chim. Acta 2008, 609, 113-119.

6. Lord, H.; Pawliszyn, J. Evolution of solid-phase microextraction technology. J. Chromatogr. A 2000, 885, 153-193.

7. LaGrega, M.D.; Buckingham, P.L.; Evans, J.C. Hazardous Waste Management; McGraw-Hill, Inc.: New York, NY, USA, 1994.

8. Stenzel, A.; Endo, S.; Goss, K.W. Measurements and predictions of hexadecane/air partition coefficients for 387 environmentally relevant compounds. J. Chromatogr. A 2012, 1220, $132-142$.

9. Zhao, H.; Zhang, Q.; Chen, J.; Xue, Z.; Liang, X. Prediction of octanol-air partition coefficients of semivolatile organic compounds based on molecular connectivity index. Chemosphere 2005, 59, 1421-1426.

10. Hong, H.; Wang, L.; Han, S. Prediction adsorption coefficients $\left(K_{\mathrm{oc}}\right)$ for aromatic compounds by HPLC retention factors $(K)$. Chemosphere 1996, 32, 343-351.

11. Nirmalakhandan, N.N.; Speece, R.E. Prediction of aqueous solubility of organic chemicals based on molecular structure. Environ. Sci. Technol. 1988, 22, 328-338.

12. Nirmalakhandan, N.N.; Speece, R.E. QSAR model for predicting Henry's constant. Environ. Sci. Technol. 1988, 22, 1349-1357.

13. Pawliszyn, J. Solid Phase Microextraction-Theory and Practice; Wiley-VCH: New York, NY, USA, 1997. 
14. Xia, X.R.; Baynes, N.A.; Monteiro-Riviere, N.A.; Riviere, J.E. A system coefficient approach for quantitative assessment of the solvent effects on membrane absorption from chemical mixtures. SAR QSAR Environ. Res. 2007, 18, 579-593.

15. Silbey, R.J.; Alberty, R.A.; Bawendi, M.G. Physical Chemistry, 4th ed.; Wiley: New York, NY, USA, 2005.

16. DiFilippo, E.L.; Eganhouse, R.P. Assessment of PDMS-water partition coefficients: implications for passive environmental sampling of hydrophobic organic compounds. Environ. Sci. Technol. 2010, 44, 6917-6925.

17. Avila, M.A.S.; Breiter, R. Estimating the DMS-coated SPME-fibre/water- and fibre/gas-partition coefficients of chlorinated ethenes by headspace-SPME. Chromatographia 2007, 66, 369-376.

18. Kier, L.B.; Hall, L.H. Molecular Connectivity in Structure-Activity Analysis; Wiley: Chichester, UK, 1986.

19. Endo, S.; Droge, S.T.J.; Goss, K.U. Polyparameter linear free energy models for polyacrylate fiber-water partition coefficients to evaluate the efficiency of solid-phase microextraction. Anal. Chem. 2011, 83, 1394-1400.

20. Sprunger, L.; Proctor, A.; Acree, W.E., Jr.; Abrahamb, M.H. Characterization of the sorption of gaseous and organic solutes onto polydimethyl siloxane solid-phase microextraction surfaces using the Abraham model. J. Chromatogr. A 2007, 1175, 162-173.

21. Goss, K.U. Predicting equilibrium sorption of neutral organic chemicals into various polymeric sorbents with COSMO-RS. Anal. Chem. 2011, 83, 5304-5308.

22. Horvath, A.L. Halogenated Hydrocarbons; Dekker: New York, NY, USA, 1982.

23. Randic, M. On characterization of molecular branching. J. Am. Chem. Soc. 1975, 97, 6609-6615.

24. Hansch, C.; Vittoria, A.; Silipo, C.; Jow, P.Y.C. Partition coefficients and the structure-activity relationship of the anesthetic gases. J. Med. Chem. 1975, 18, 546-548.

(C) 2014 by the authors; licensee MDPI, Basel, Switzerland. This article is an open access article distributed under the terms and conditions of the Creative Commons Attribution license (http://creativecommons.org/licenses/by/3.0/). 